Nuclear Instruments and Methods 178 (1980) 331-343

(c) North-Holland Publishing Company

\title{
A SILICON SURFACE BARRIER MICROSTRIP DETECTOR DESIGNED FOR HIGH ENERGY PHYSICS
}

\author{
E.H.M. HEIJNE, L. HUBBELING, B.D. HYAMS, P. JARRON, P. LAZEYRAS, F. PIUZ, J.C. VERMEULEN \\ and A. WYLIE \\ CERN, Geneva, Switzerland
}

Received 4 August 1980

A silicon microstrip detector was manufactured using the surface barrier technique. It has 100 strips at $200 \mu \mathrm{m}$ pitch and it is $400 \mu \mathrm{m}$ thick. To each strip a fast current sensitive preamplifier is connected so that minimum ionizing particles can be detected on single strips. The signals have a duration of $40 \mathrm{~ns}$ and are processed using standard equipment. A description is given of the results which were obtained in two high energy particle beams at CERN.

\section{Introduction}

Silicon detectors have already been used in high energy physics as a target for coherent interactions in a series of experiments by Bellini et al. [1]. Yuan [2] was probably the first to use them for detection of $\mathrm{GeV}$ particles and, more recently, Esbensen et al. [3] measured energy deposition by $2-15 \mathrm{GeV} / c$ protons, pions and kaons under channelling and random incidence. But in general, solid state detectors have been used very little for high energy physics experiments because they cannot provide the large detecting area which is required in common spectrometers. While it is still true that multiwire proportional chambers (MWPC), drift chambers or scintillation counters offer the best solution for a large area, a need is now felt for thin particle detectors very near to the interaction region, with high precision, high rate and high multiplicity capability, which is difficult to meet with existing detectors. Especially the study of the very short-lived charmed particles will profit from an electronic detector which enables detection and track reconstruction on micron scale, in the target region. The nuclear emulsion technique is unsurpassed in spatial resolution, but does not allow high event rates or electronic triggering. Recent emulsion experiments use a hybrid apparatus, in which external detectors identify possibly interesting interactions inside the emulsion $[4,5]$. But even so, improvement of the spatial resolution of these detectors would be very welcome.

We decided to study the properties of a silicon microstrip detector and associated electronics for the purpose of detecting minimum ionizing particles. We explain the design considerations for the detector and electronics, and report on measurements performed with four prototype silicon surface barrier microstrip detectors in $10 \mathrm{GeV} / c$ and $175 \mathrm{GeV} / c$ hadron beams at CERN. The practicability of a silicon microstrip detector is demonstrated, and possible applications are discussed. We expect that, when state of the art miniaturization and integration are incorporated, a very promising detector can be obtained for particle rates in excess of $10^{8} \mathrm{~s}^{-2} \mathrm{~cm}^{-2}$, with spatial resolution of the order of $10 \mu \mathrm{m}$ and which can be operated inside a vacuum chamber.

\section{The position sensitive microstrip detector}

Position sensitive strip detectors were made already in the early days of the silicon detector. Strip widths from $0.5 \mathrm{~mm}[6,7]$ to $1 \mathrm{~mm}[8-10]$ are reported. A checker board detector $[11,12]$ with strips of $1.37 \mathrm{~mm}$ oriented orthogonally on the front and the back side of the wafer has been available commercially. But in nuclear physics the strip detectors have been largely abandoned in favour of position sensitive detectors using charge division read-out [13]. Radeka recently proposed [14] to use resistive charge division in combination with a centroid finding method for position sensitive detection of high energy particles. The main problem in the realization of such a detector lies in the homogeneity required 
for the resistive layer, but also the detector must be rather thick to obtain a sufficient signal to noise ratio. We have returned to the thin strip detector design with very fine strips $(20-140 \mu \mathrm{m})$. For the first prototypes we have chosen for 100 strips of 140 $\mu \mathrm{m}$ wide, $30 \mathrm{~mm}$ long and a $200 \mu \mathrm{m}$ pitch. The sensitive area of this detector is $20 \times 30 \mathrm{~mm}^{2}$, which is suitable for most hadron beams above $50 \mathrm{GeV}$, both in the beam and just behind a target.

\subsection{Detector thickness}

The thickness of the detector, apart from mechanical considerations, is mainly determined by the required input charge for the preamplifier. In the absence of internal multiplication, this is the charge which is generated by the ionization of the particle in the silicon. The ionization is proportional to the energy loss, which is described by a Landau distribution. The most probable energy deposition in a thickness of $100 \mu \mathrm{m}$ of Si by a minimum ionizing particle is 29 $\mathrm{keV}$, which results in the formation of $\sim 8000$ electron-hole pairs, or a charge of $1.3 \mathrm{fC}$. The prototype detectors have a thickness of $400 \mu \mathrm{m}$ so that the most probable signal is $5.2 \mathrm{fC}$, corresponding to $\sim 120 \mathrm{keV}$ energy deposition. But when the charge is distributed over two strips, e.g. for inclined particle tracks, the signal on either strip may be much less. With the present preamplifier performance, as discussed in the next section, the signal/noise ratio obtained with 400 $\mu \mathrm{m}$ thickness is largely sufficient for room temperature operation.

\subsection{Detector construction}

The surface barrier diode structure was chosen because it is a straightforward technique for silicon detectors. But, unfortunately, surface barrier detectors are rather delicate. Planar technology as used in integrated circuit fabrication would enable a more

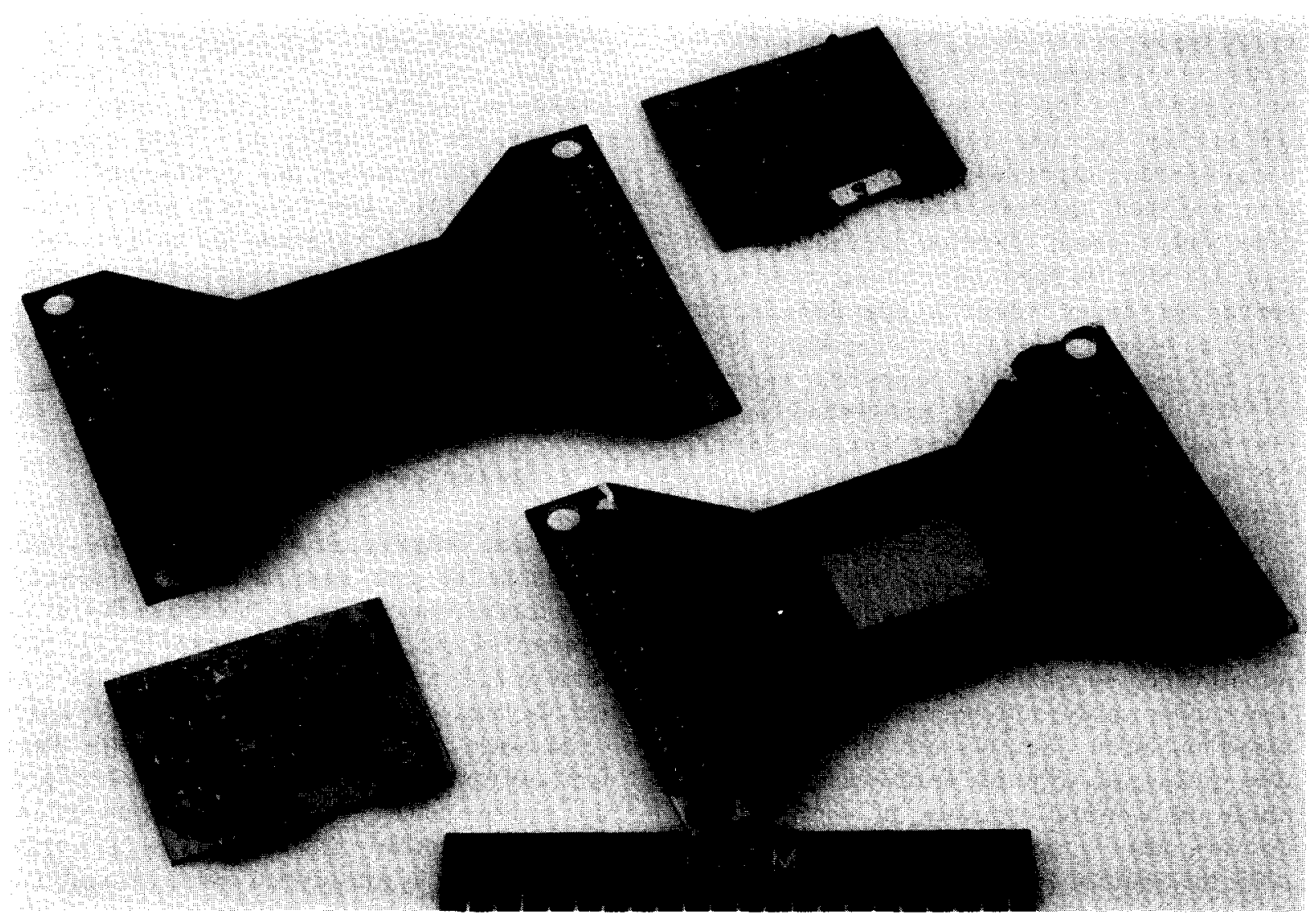

Fig. 1. Silicon surface barrier microstrip detectors, with strips on the rectifying side (above) or on the ohmic contact side (below). Also shown are two modules of the dual current sensitive preamplifier. 
rugged device with better precision. An extensive development program, however, is required to obtain microstrip particle detectors by a planar technique, but this may be worthwhile if spatial resolutions of below $20 \mu \mathrm{m}$ are sought.

Silicon wafers of $24 \times 30 \mathrm{~mm}^{2}$ are prepared as usual. After the final etching, the silicon wafer is glued with an epoxy resin into a fiber glass printed circuit board, which already contains the contacts in the form of gold plated copper strips. The microstrip pattern is then obtained by evaporation of the metal through a mask which is carefully aligned with the contacts. The mask is a stretched $\mathrm{Cu}-\mathrm{Be}$ foil of $50 \mu \mathrm{m}$ thick in which the strips are etched by standard photo-engraving procedures. Although the evaporated strips are rather fragile where they make the contact across the epoxy edge, only $\sim 10 \%$ of the strips were unconnected. As will be described in sect. 4 , particles crossing an unconnected strip are detected on the adjacent strips and therefore only a loss in precision occurs, but nearly no loss in efficiency. In making the contacts this way, less work is involved, and a very thin detector package is obtained which facilitates stacking in several layers. The resistivity of the n-type silicon used was $\sim 8000 \Omega \mathrm{cm}$ so that the detector is totally depleted at $90 \mathrm{~V}$ reverse bias. Normally the detectors are operated at $110 \mathrm{~V}$ reverse bias, but the behaviour was checked from $10 \mathrm{~V}$ to $170 \mathrm{~V}$ bias. Above $170 \mathrm{~V}$ the noise increased rapidly with increasing reverse leakage current. At $110 \mathrm{~V}$ $\left(20^{\circ} \mathrm{C}\right)$ the leakage current was around $1 \mu \mathrm{A}$ for the aluminium strip detectors and around $2 \mu \mathrm{A}$ for the gold strip detectors, for the whole $6 \mathrm{~cm}^{2}$ area. The current on single strips is about $1 / 100$ of this. The measured diode capacitance of the strips at total depletion was between 12 and $15 \mathrm{pF}$, including the leads.

The four prototype detectors were manufactured in collaboration with Enertec/Schlumberger (France). Two have gold strips on the rectifying side and a continuous aluminium ohmic back contact, the other two have aluminium strips at the back and a continuous gold surface barrier. In fig. 1 a picture is shown of both the gold strip detector and the aluminium strip detector, together with two dual preamplifier modules. The main difference between the two detectors is that the aluminium strip detector must be totally depleted to obtain position sensitive, fast signals, whereas the gold strip detector is already useable at $10 \mathrm{~V}$ reverse bias, although the signal height is much reduced. In fig. 2 the signal height for minimum ionizing particles is plotted as a function of reverse bias for both types of microstrip detector. We used the fast preamplifier described in the next section.

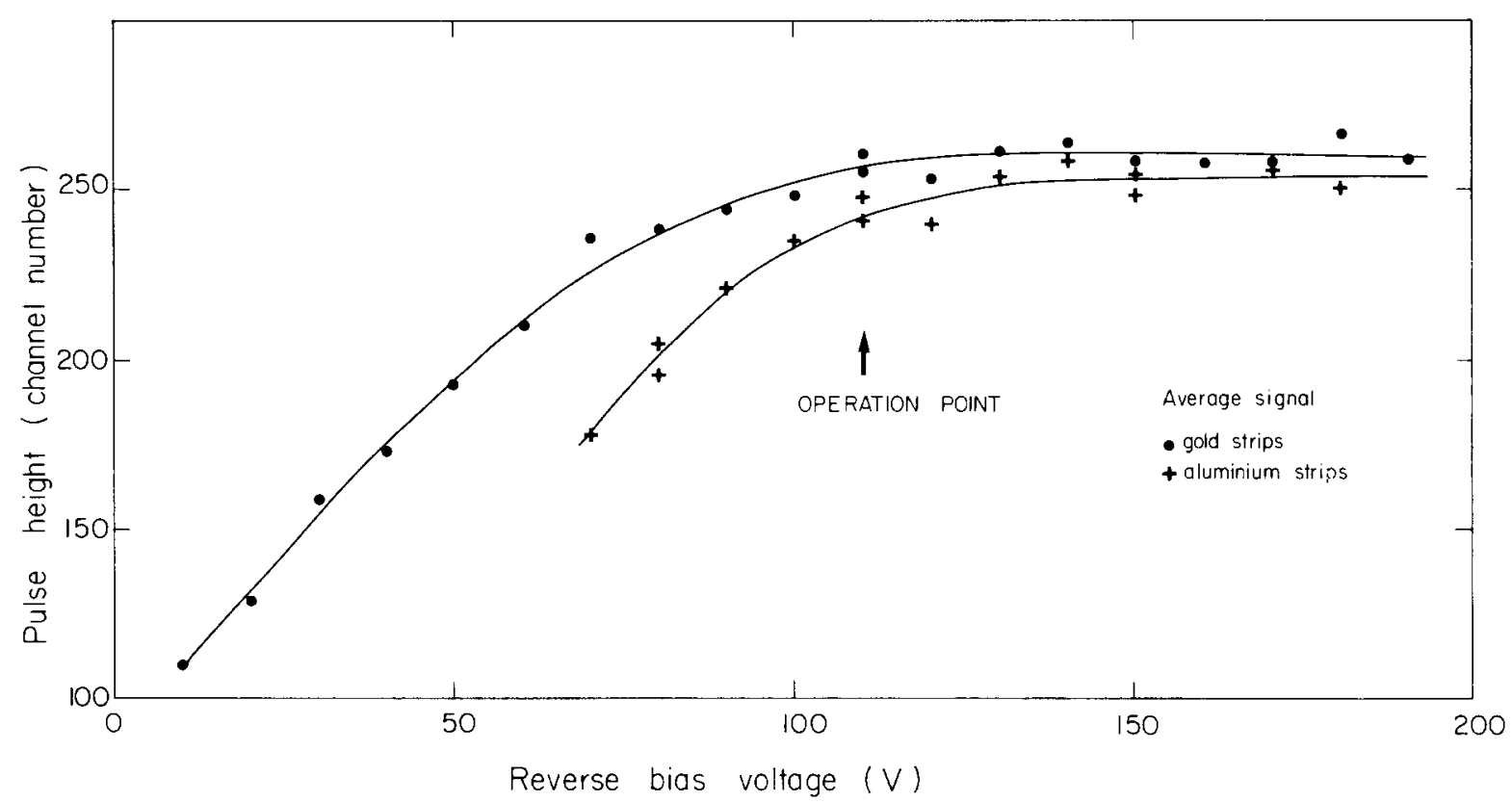

Fig. 2. Evolution of the pulse height as a function of reverse bias voltage for the aluminium strip detector and the gold strip detector. 


\section{Signal processing and fast preamplifier}

The preamplifier design and the signal processing will depend on the particular needs of the experiment. In nuclear physics the excellent energy resolution of solid state detectors is generally of highest importance, requiring low-noise performance of the amplifier. In the present work we wished to emphasize the high rate capability of the silicon detector and therefore a fast, current sensitive, preamplifier with a current gain of 800 was designed, which can be used in conjunction with a standard linear amplifier.

\subsection{The detector signal}

The maximum transit times of the electrons and the holes which are generated by the particle depend on the applied reverse bias voltage, and for a $400 \mu \mathrm{m}$ thick detector at $100 \mathrm{~V}$ (i.e. $2500 \mathrm{~V} \mathrm{~cm}^{-1}$ ) they are about $10 \mathrm{~ns}$ and $25 \mathrm{~ns}$ respectively. The tiny amount of charge $(\sim 5 \mathrm{fC})$ represents a current signal of $\sim 0.25$ $\mu \mathrm{A}$ at the input of the preamplifier. The rise time of this current signal is shorter than the maximum transit time, because for minimum ionizing particles the charge is evenly distributed through the detector thickness. A thin silicon detector therefore has, in principle, a fast response. Long shaping time constants are necessary if low noise is important, but for minimum ionizing particles the main signal variations originate from the fluctuations in deposited energy, as given by the Landau distribution. In $100 \mu \mathrm{m}$ of silicon the most probable deposit is $\sim 29 \mathrm{keV}$ but the average deposit is $34 \mathrm{keV}$ (see e.g. ref. 3). It should be noted that the energy deposition in a thin detector is less than the theoretically predicted energy loss in an infinite absorber, which is $40 \mathrm{keV}$ at the ionization minimum.

\subsection{The preamplifier}

Among the three possible types: charge, current or voltage sensitive amplifiers we have chosen to develop in association with the microstrip detector a low noise, wideband current sensitive preamplifier. Reasons for this choice are:

a) to make maximum use of the fast signal characteristics of the thin silicon detector;

b) a fast signal is suitable for treatment with existing standard NIM and CAMAC equipment;

c) no filter amplifier is needed, as is the case with a charge sensitive or a voltage sensitive preamplifier [15];

d) at high frequency, microwave low noise bipolar transistors can be used instead of field effect transistors;

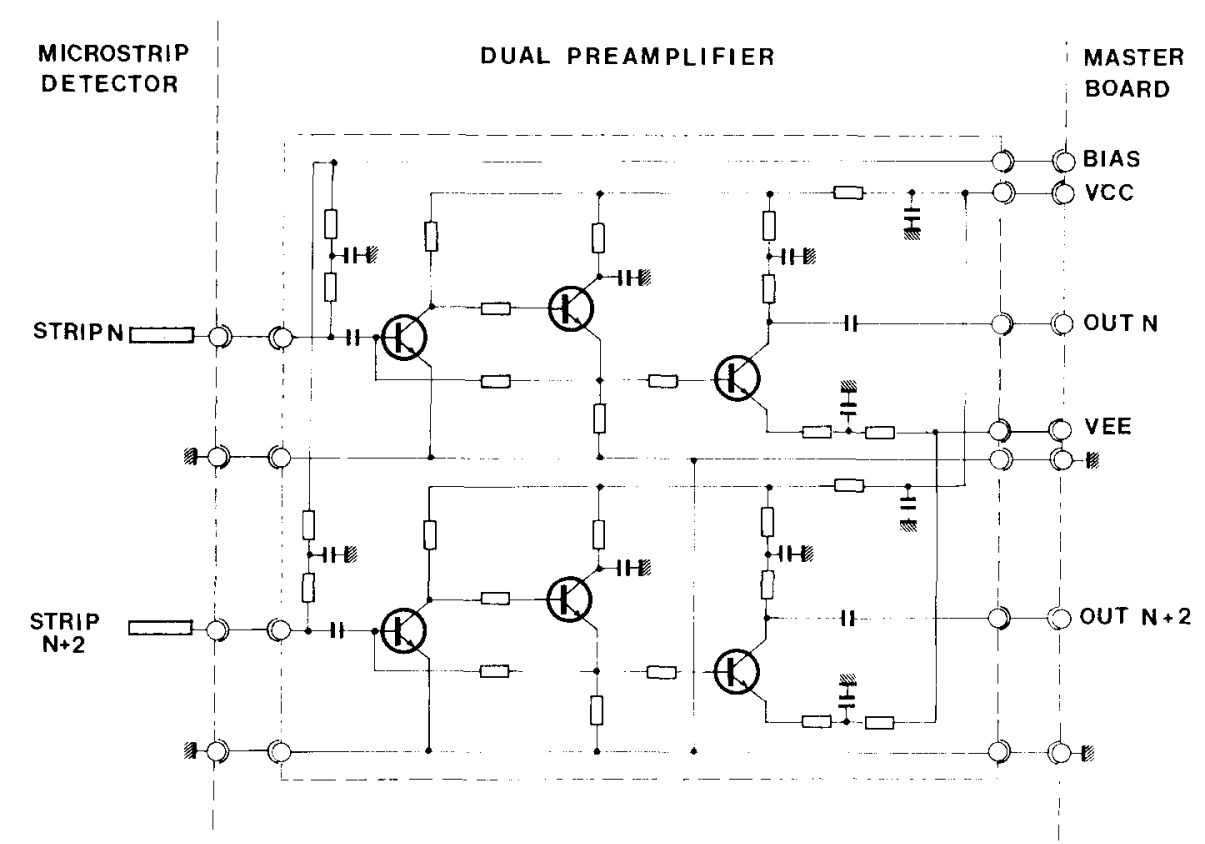

Fig. 3. Circuit diagram of the dual current sensitive preamplifier. 
e) a better immunity against cross talk is obtained with a low input impedance current sensitive preamplifier.

The use of bipolar instead of field effect transistors is advantageous in several respects. It allows a simple circuit at lower cost, a high packing density and lower power consumption. The drain current of a FET can be $10 \mathrm{~mA}$, and for a compact amplifier arrangement around a microstrip detector with 1000 channels the heat generated would be a severe problem.

The noise versus bandwidth optimization led to a feedback pair amplifier, also called a transimpedance amplifier. The design is such that the noise from the three principal sources is minimized. These are the shot noise on the base and collector currents in the input transistor, the thermal noise from the feedback resistance and the series voltage noise from the base resistance. This preamplifier, as shown in fig. 3 , is
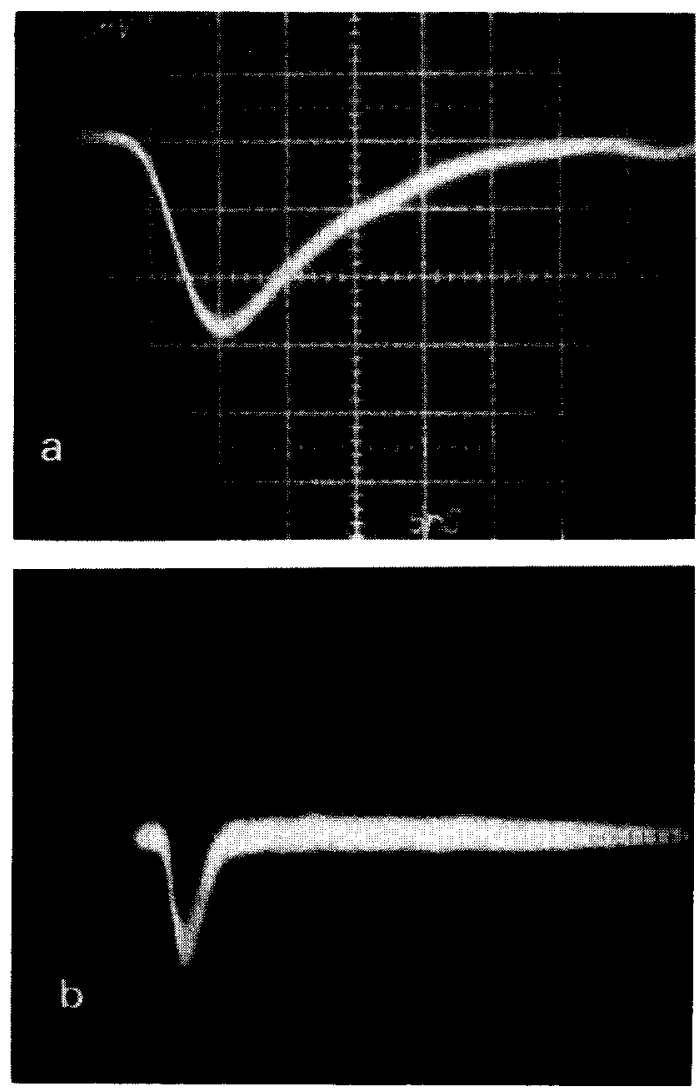

Fig. 4. Amplified output signal of the preamplifier (a) with test pulse of $10 \mathrm{mV}$ on $0.75 \mathrm{pC}$; horizontal $5 \mathrm{~ns}$, vertical $50 \mathrm{mV}$ (b) connected to microstrip detector in the beam; horizontal $50 \mathrm{~ns}$, vertical $100 \mathrm{mV}$.
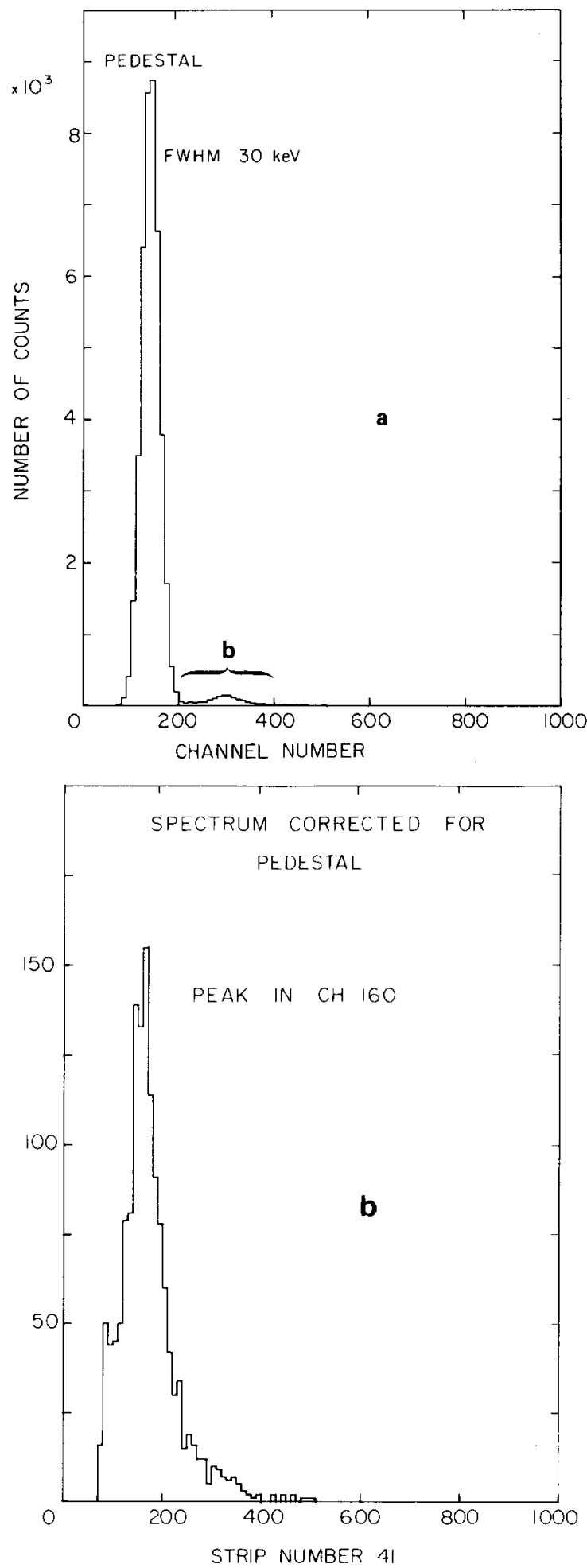

Fig. 5. (a). Pulse height spectrum of signals from a microstrip in the $10 \mathrm{GeV} / c$ test beam. The width of the pedestal gives the noise. (b) Enlargement of the Landau distribution after pedestal correction. 
very simple and rather cheap. Components cost is $\sim 20 \mathrm{SF}$. To obtain a high packing density, several preamplifiers must be built on the same card. At present a dual version is used. A hybrid circuit will allow an even denser packing, and is easy to realize for this simple design. The input transistor has a high bandwidth, a low base-collector capacitance and a high current gain. With an input voltage of $10 \mathrm{mV}$ on $0.75 \mathrm{pF}$ the noise charge was measured with a charge integrating pulse height analyser, gated for $40 \mathrm{~ns}$. A fwhm of $0.54 \mathrm{fC}$ or $12 \mathrm{keV}$ silicon equivalent energy loss was found, corresponding to 1400 electrons r.m.s. The signal is shown in fig. 4a.

The performance in connection with the microstrip detector is illustrated in fig. $4 \mathrm{~b}$. The output signal into $50 \Omega$ is $5 \mathrm{mV}$ for a minimum ionizing particle. The integrated charge in this signal is proportional to the energy deposited in the detector. The rise time is $\sim 15 \mathrm{~ns}$ for a signal duration of $40 \mathrm{~ns}$. A pulse height spectrum obtained in the beam of 10 $\mathrm{GeV} / c$ particles is shown in fig. 5 . The width of the pedestal noise distribution (PND) indicates a fwhm noise of $30 \mathrm{keV}$. In fig. $5(\mathrm{~b})$ the pedestal correction is applied as follows. The origin is shifted to the position of the mean value of the PND, and all signals which are less than three times one standard deviation of the PND are eliminated.

If, in some application of the microstrip detector much lower noise is required, a charge sensitive preamplifier with FET input stage in the usual configuration should perform better, at the expense of a loss in speed and more power consumption.

\subsection{Further signal processing}

No line driver was included in the electronics design, therefore the output signals are sent via $10 \mathrm{~m}$ of coaxial cable to standard linear amplifiers (LRS 612 AM). A double output on this amplifier permits simultaneous analog and logical processing. The analog signal goes to a CAMAC ADC (LRS 2249) which integrates the charge signal during a $60 \mathrm{~ns}$ gate, and which is read-out subsequently by a computer data acquisition program. The signal from the second output goes to a discriminator and the resulting logical signal is used for fast scaling, coincidences etc.

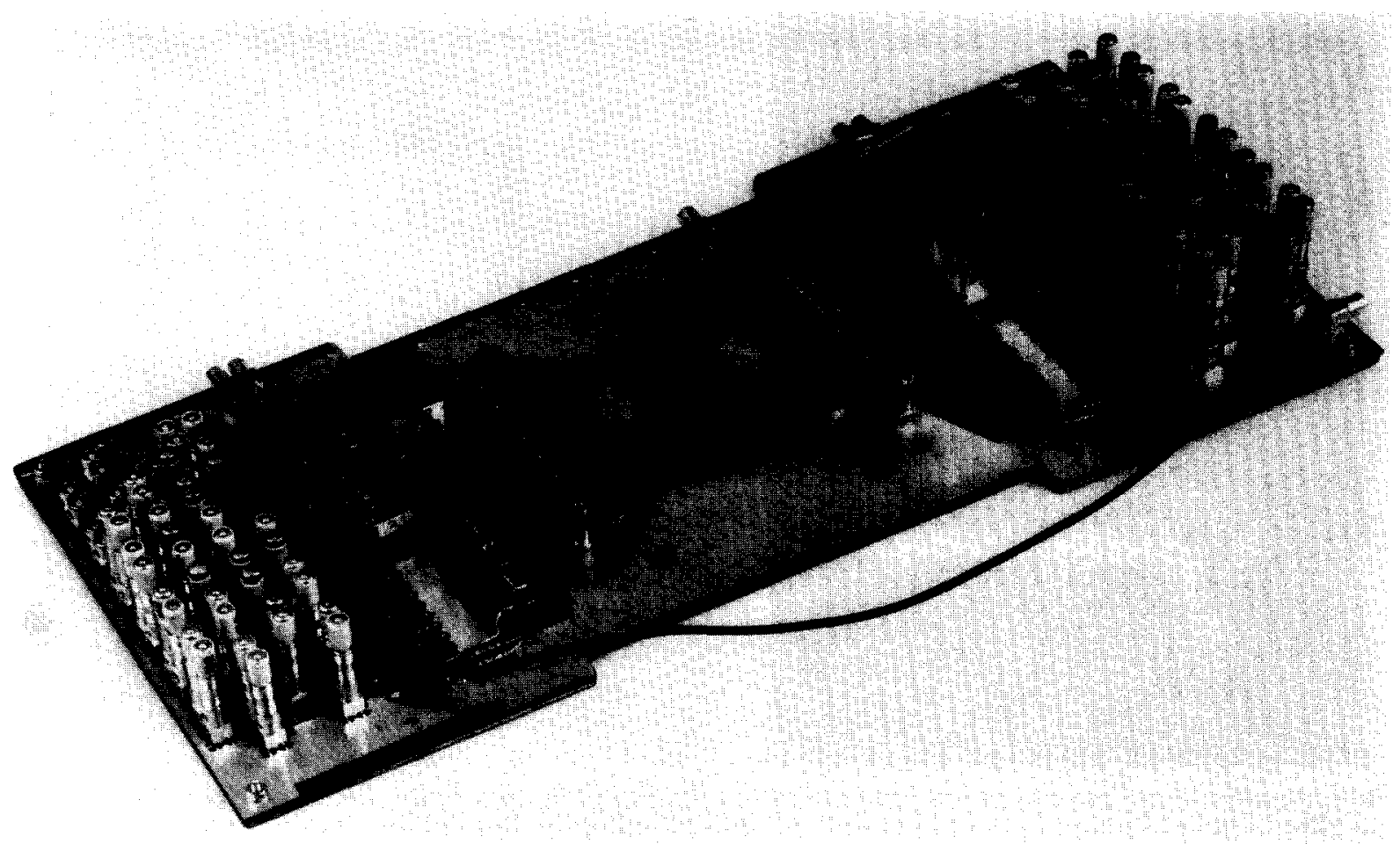

Fig. 6. The microstrip detector placed together with some amplifiers on the test board, which can be positioned in a beam. 


\subsection{Cross talk}

Some precautions were taken to reduce the electrical cross talk between strips, and in fact for minimum ionizing particles this electronic cross talk on adjacent strips is less than $10 \%$ and buried in the noise. On the other hand, simultaneous signals on adjacent strips are observed in a small fraction of the events. This phenomenon of "double hits" is discussed in sect. 5.3.

The capacitance between the strips causes a mutual influence between amplifiers, and creates apart from cross talk also oscillation problems. In order to avoid these problems the amplifiers for adjacent strips were placed on opposite sides as is shown in the picture in fig. 6. Also electrical shielding is provided all around the detector and the amplifiers. The test board shown in fig. 6 was designed to enable close stacking of 2, 3 or 4 similar microstrip detectors. Because the detector is plugged in with pins, a change of detector in the beam-line only took $10 \mathrm{~min}$. Also the preamplifiers can be easily replaced.

\section{Performance of the detector in a $10 \mathrm{GeV} / \mathrm{c}$ test beam}

The scattering of low energy beta particles or gammas from radioactive sources leads to signals on several adjacent strips, and therefore a microstrip detector can only be realistically tested in a beam of high energy particles. In a test beam of $10 \mathrm{GeV} / c$ pions and protons the "aluminium" microstrip detector was placed between wire chambers with centre of gravity read-out on the cathode strips [16]. The setup is sketched in fig. 7. The trigger was given by the scintillators $\mathrm{S} 1$ and $\mathrm{S} 2$ and sometimes it also included strip 48 from the detector itself. From this trigger was derived a gate of $60 \mathrm{~ns}$ on the detector signals, which were read out together with those from the

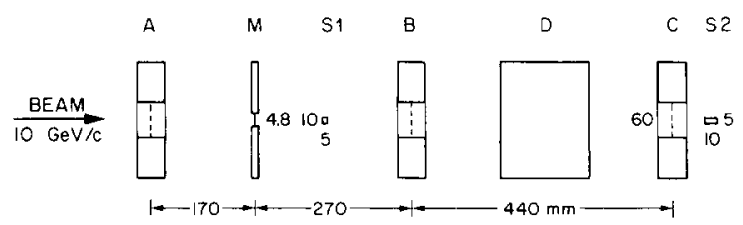

Fig. 7. Situation of the microstrip detector $M$ between wire chambers with centre of gravity read-out A, B and C. Scintillators $S 1$ and $S 2$ give a trigger and the gate for read-out. $\mathrm{D}$ is a drift chamber, which was not used. centroid chambers after analog to digital conversion. From the measured particle positions in chambers $A$ and $\mathrm{B}$, the position on detector $\mathrm{M}$ can be predicted.

With the trigger (S1 $\cdot \mathrm{S} 2 \cdot$ strip 48) 18502 events were recorded. The pulse height spectra for this trigger are shown in fig. 8a. The energy deposition spectrum from strip 48 , the trigger strip, follows a Landau distribution, but with a tail towards low energy. If only those events are selected, which have no simultaneous hit on a neighbouring strip ("single hits"),

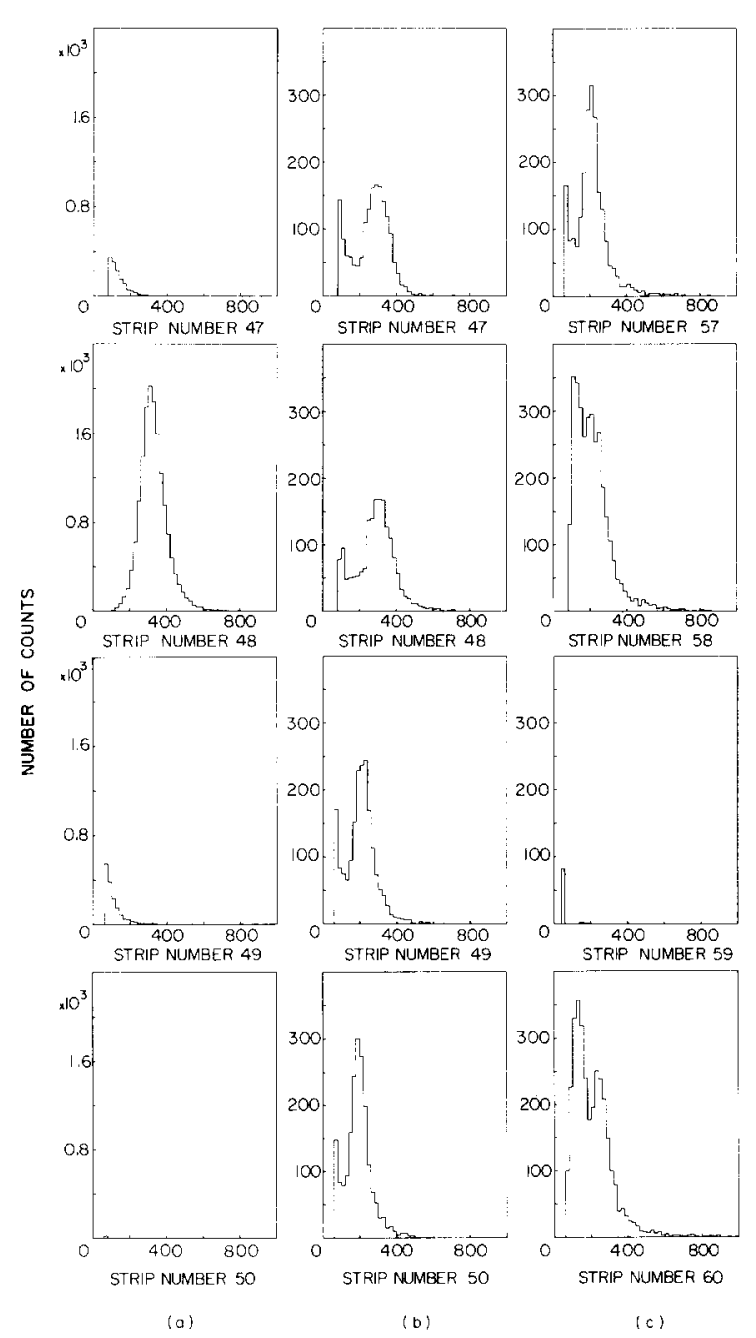

Fig. 8. Pulse height spectra for a few microstrips:

(a) Strip 48 is in the trigger. Adjacent strips show a number of low signals, other strips have nearly no simultaneous hits. (b) Only the scintillators S1 and S2 give the trigger. All strips show a nearly identical spectrum.

(c) Strips 58,59 and 60 have particular behaviour, because strip 59 has a broken contact. Same trigger as in (b).

In all these spectra the pedestals were eliminated. 


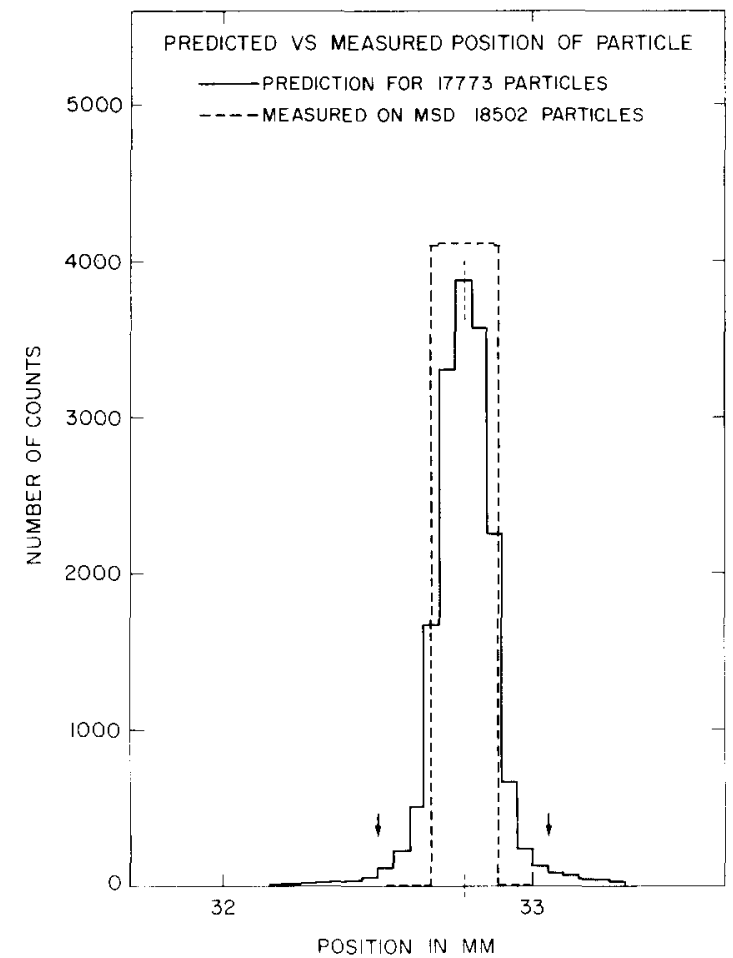

Fig. 9. Distribution of the predicted position of the particles, calculated from the signals of wire chambers A and B, compared with the measured position on the microstrip detector M. $84 \%$ of the events are single hits and $16 \%$ double hits. this low energy tail disappears. A hit is defined as a signal which exceeds the mean plus three standard deviations of the PND (see 3.2).

In $96 \%$ of the events the track could be reconstructed with the information from chambers $A$ and B. The distribution of the predicted points is shown in fig. 9 together with the actual measurements. The strip is either hit or not and therefore the distribution is square in this case. $7 \%$ double hits occurred between strips 47 and 48 and $8 \%$ between 48 and 49. Assuming homogeneous distribution of the particles over the detector surface area, and also assuming that double hits are caused by particles in between strips, the region where particles give a single hit is $184 \mu \mathrm{m}$ wide and double hits occur in a $16 \mu \mathrm{m}$ zone. The sum of the signals in double hits is slightly higher than the average value for single hits. More detailed results on double hits are discussed in sect. 5.3. Triple hits occur in less than $1 \%$ of the events. These can be explained as particles with an accompanying delta electron or a converting gamma. Altogether, the microstrip detector with $200 \mu \mathrm{m}$ pitch gives a slightly better result than the centroid chambers which have a sigma of only $\sim 80 \mu \mathrm{m}$ of the distribution of the predicted positions.

When only S1 and S2 generate the trigger, events are nearly homogeneously distributed over the 24

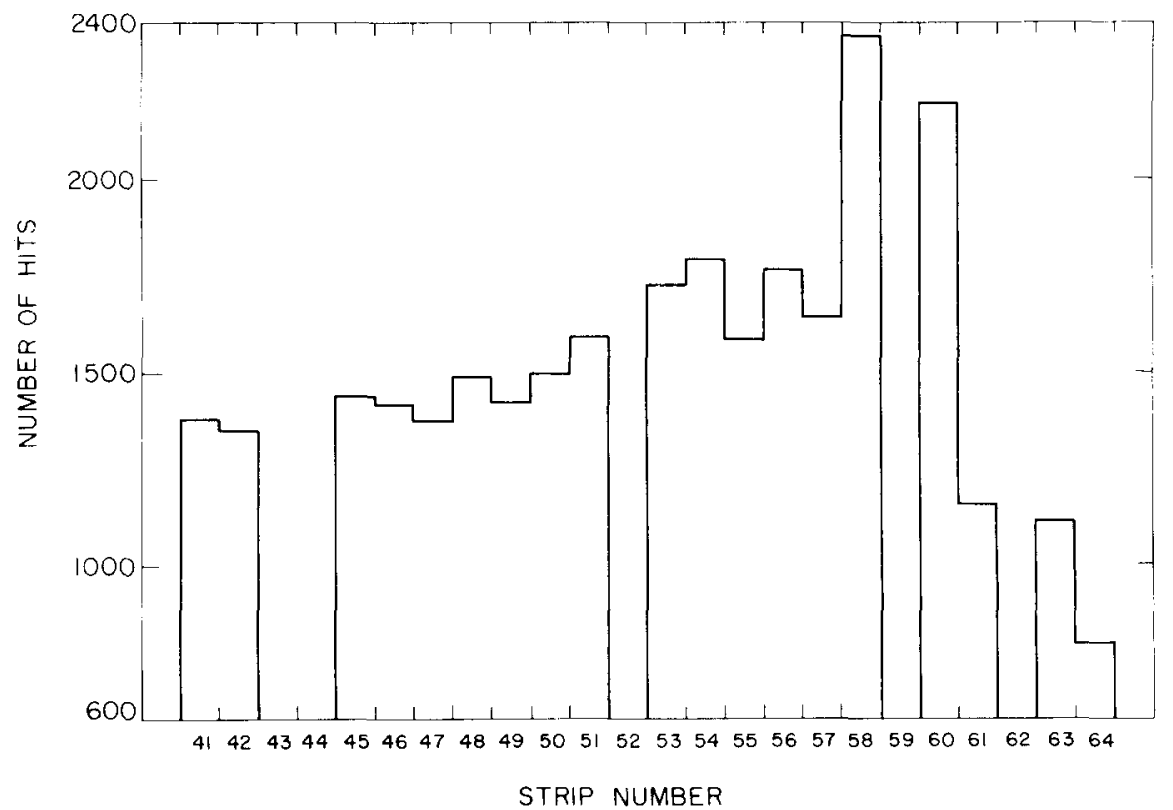

Fig. 10. Hit distribution over 24 strips in the $10 \mathrm{GeV}$ beam. Amplifiers on strips 43, 44, 52 and 62 were not working. Strip 59 has a broken contact, but its signals are collected on either 58 or 60 . 
strips, as is shown in fig. 10. Exceptions are the strips $43,44,52$ and 62 , for which the preamplifiers were not working correctly. Some spectra are shown in fig. $8 \mathrm{~b}$. They are corrected for the pedestal, which in the original data was adjusted to be around channel 150. All spectra show a Landau distribution, with a low energy tail, sometimes with a remnant of the PND. Also here, the double hits are responsible for this low energy tail. A special case is presented by strip 59 which happens to be unconnected. The signals of particles passing through this strip are collected on the adjacent strips 58 and 60 , as can be seen from the count distribution in fig. 10. The signal height is reduced however, as is shown in the spectra in fig. 8c.

\section{Test in $175 \mathrm{GeV} / c$ beam}

During a 10 day run the microstrip detector was positioned in between the beam hodoscopes of a CERN SPS experiment (NA 11), and the main exper- iment trigger provided a gate for $\mathrm{ADC}$ read-out on 40 strips. Only single beam particles are recorded with this trigger. For a dedicated time, however, the detector was placed $24 \mathrm{~mm}$ downstream of a special target, and an interaction trigger was given by a small scintillator just behind the microstrip detector.

\subsection{Beam profile}

The same aluminium strip detector as used in the $10 \mathrm{GeV} / c$ beam was at the focus of the beam, which has a size of $2 \times 18 \mathrm{~mm}^{2}$ and contains $\sim 3 \times 10^{6}$ pions, with a $1.8 \mathrm{~s}$ spill time every $10.8 \mathrm{~s}$. The electronic layout was as described in sect. 3.3 with the amplifier gains adjusted such that the signal of the most probable energy deposition corresponds to channel $\sim 250$ in the pulse height spectrum. Parallel to the gated analog read-out, the signals from 36 strips were passed to discriminators and fast scalers. The microstrip detector functioned as a precise onedimensional beam profile monitor. In fig. 11 the profile measured in one burst is shown. The useful beam

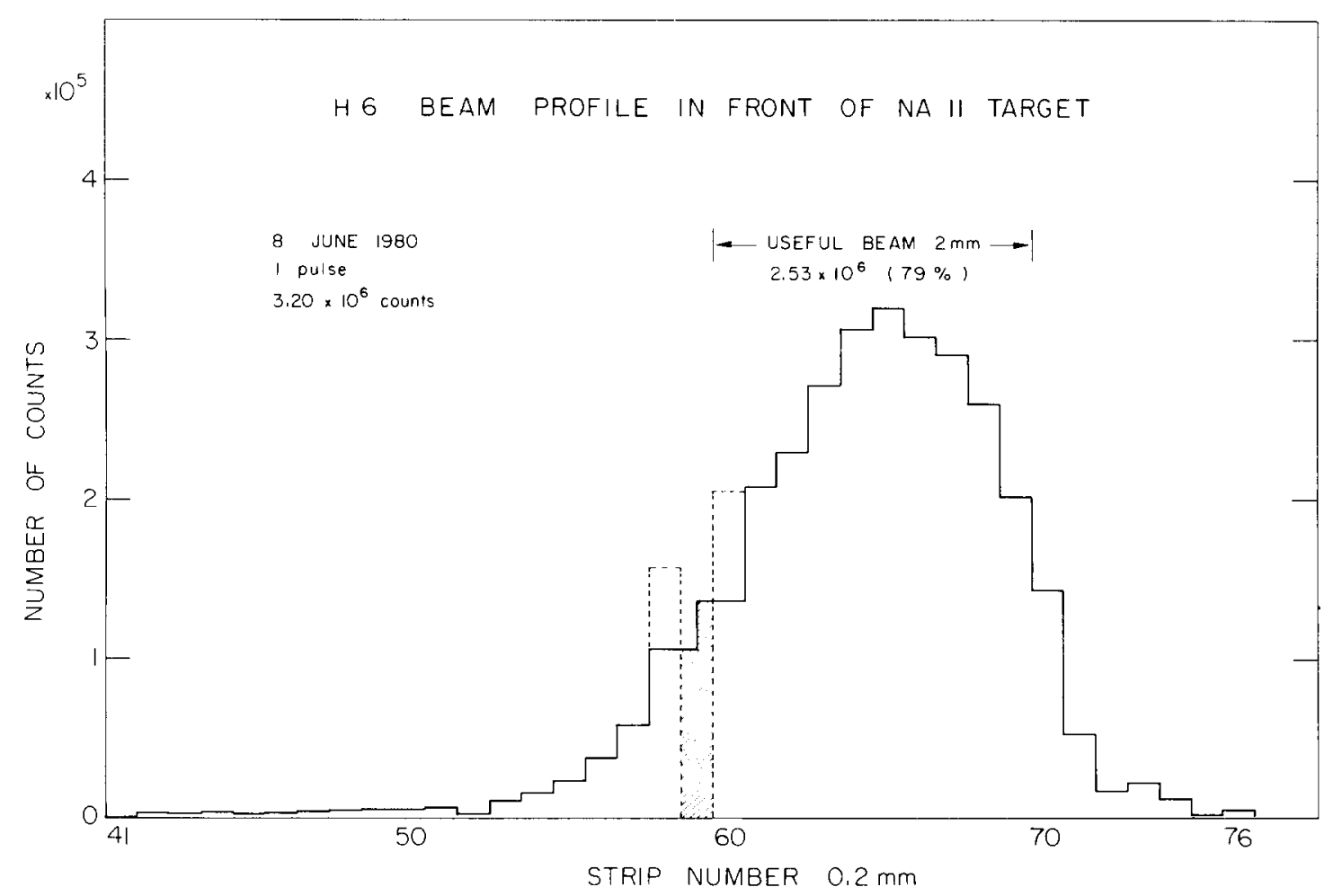

Fig. 11. Vertical profile of the $175 \mathrm{GeV} / c$ hadron beam in front of the NA11 target, measured with the microstrip detector. In one beam pulse on all strips a total of $3.20 \times 10^{6}$ particles was counted, whereas the NA 11 beam counter recorded $3.18 \times 10^{6}$ particles. Each strip is $200 \mu \mathrm{m}$ high and $30 \mathrm{~mm}$ long. Strip 59 is the broken one. 
has $2 \mathrm{~mm}$ height (strips 60-69), and is easily contained in the $20 \times 30 \mathrm{~mm}^{2}$ sensitive area.

\subsection{Efficiency}

As the microstrip detector was placed between the NA1 1 beam hodoscopes, beam tracks can be selected which pass through the detector, and these should then be found by the detector. On 9745 tracks only 39 were not found, partly due to the fact that strip 59 has a broken contact and therefore the particles are measured on adjacent strips but at reduced pulse height. The pedestal correction which is applied may then cause some loss. The resulting efficiency is at least $99.6 \%$.

\subsection{The double hits}

Also in this test simultaneous hits on adjacent strips were recorded in $10-15 \%$ of the beam particle events. To study this phenomenon in more detail, the detector was rotated by $45^{\circ}$ and the pulse height spectra are compared to those for normal incidence. The situation is sketched in fig. 12 . Under $45^{\circ}$ incidence either two (c) or three cells (d) of $200 \mu \mathrm{m} \mathrm{X}$ $400 \mu \mathrm{m}$ are crossed by a particle. The central cell, which has the highest signal should contain $1 / \sqrt{ } 2$ times the signal at normal incidence. In fig. 13 the

SILICON SURFACE BARRIER MICROSTRIP DETECTOR

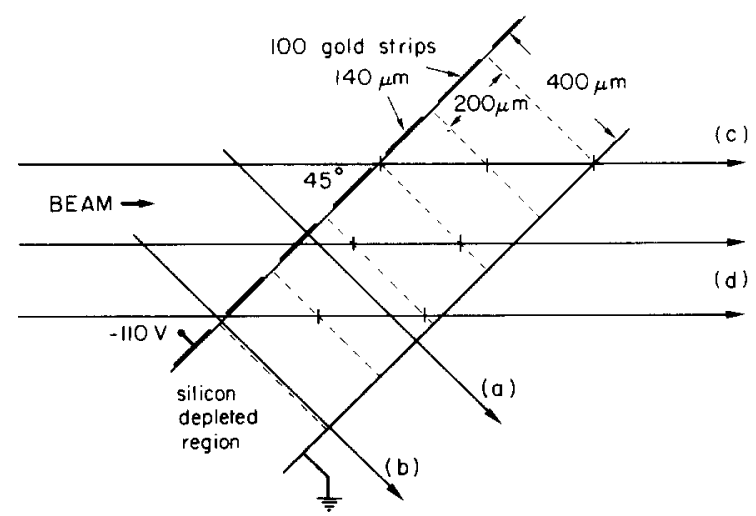

Fig. 12. Schematic drawing of particles passing through the microstrip detector, which is rotated over $45^{\circ}$. Normal incidence is also indicated, particle (a) gives a single hit, whereas (b) in the interstrip zone may give a double hit. Under $45^{\circ}$ particle (c) just crosses 2 cells, whereas most particles will cross three cells (d). The central cell in case (d) always has the same signal, provided the beam is exactly parallel and the cell boundary is perpendicular to the detector surface. normal incidence spectrum (a) for strip 54 is compared to those for the maximum signal of the adjacent strips 53, 54 and 55 and for their sum. In table 1 results are given for a different strip (63), which are based on about 5000 events.

For easy comparison, the results at $45^{\circ}$ are divided by $\sqrt{ } 2$. The highest signal is obtained in case of a double hit at normal incidence (b), and nearly as much is found for the sum of the signals in a triple hit at $45^{\circ}$. The most probable signal is only $2 \%$ lower and the mean value differs by $5 \%$. A much smaller signal occurs apparently for single hits in normal incidence: it is $20 \%$ lower for the most probable signal and on the average reduced by $35 \%$. A loss of signal to neighbouring strips in this case does not seem to be the explanation because there is hardly an increase when the ADC content from the adjacent strip 62 is included in the peak height $\left(a^{\prime}\right)$. Therefore, a higher total signal is characteristic for double hits. It could be due to a physical process in the charge generation, like $\delta$-rays which reach the neighbouring cell.

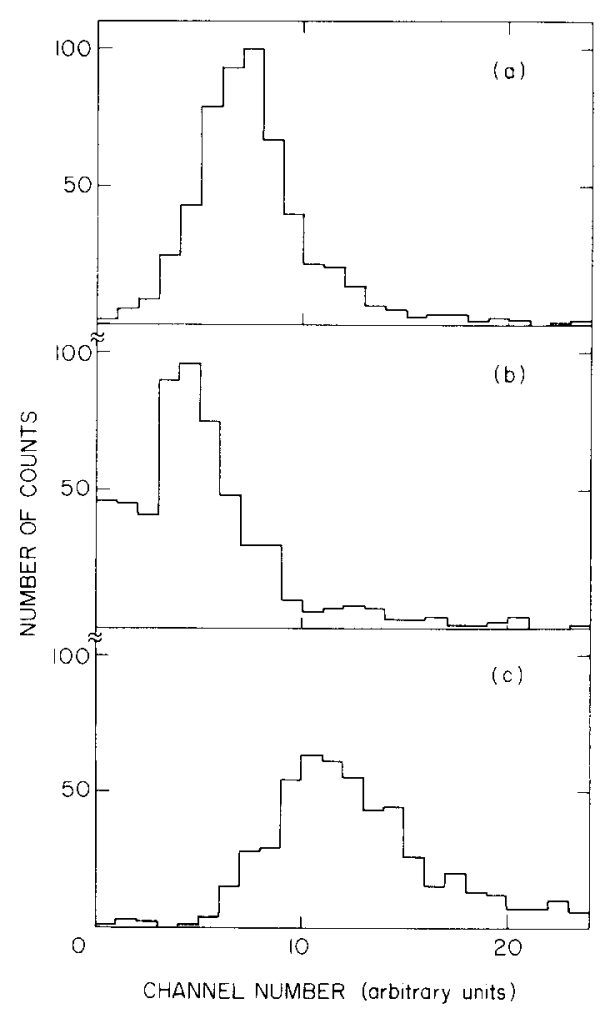

Fig. 13. Pulse height spectra for normal incidence (a) and under $45^{\circ}$; (b) is the spectrum of the central cell, (c) is the spectrum of the sum of the three cells. 
Table 1

Comparison of signal heights (channel number).

\begin{tabular}{lllll}
\hline & Event classification & Contents of histogram & $\begin{array}{l}\text { Most probable } \\
\text { signal height }\end{array}$ & $\begin{array}{l}\text { Mean value } \\
\text { of signals }\end{array}$ \\
\hline $\begin{array}{l}\text { Normal } \\
\text { incidence }\end{array}$ & 63 only & Signal on 63 & 230 & $260(\mathrm{a})$ \\
& 63 only & Sum of signal $62+63$ & 240 & $268\left(\mathrm{a}^{\prime}\right)$ \\
& $62+63$ & Sum of double hits & 275 & 350 (b) \\
$45^{\circ}$ incidence & & & & $250(\mathrm{c})$ \\
divided by $\sqrt{ } 2$ & $62+63$ & Sum of double hits & 220 & $322(\mathrm{~d})$ \\
& $62+63+64$ & Sum of three hits & 270 & $166(\mathrm{e})$ \\
\hline
\end{tabular}

\subsection{Detector in target position}

A polyethylene target replaced for a short period the usual target and the microstrip detector was placed behind it, as is shown in fig. 14. The strips were horizontal. Events were recorded in the microstrip detector and in the spectrometer, which operated without the magnetic field. Using the information of 20 drift chamber planes placed at $2.5-3.5 \mathrm{~m}$ downstream of the target, events could be reconstructed, with a $2-3 \mathrm{~mm}$ precision in the vertical position at the target of the tracks, corresponding to the $\sim 0.7 \mathrm{mrad}$ precision on their angles, not included the error due to multiple scattering. An example is shown in fig. 14. Nine tracks were found in the drift chambers, of which six, (1)-(6), point back to a vertex near the target position. Two tracks come from a space point, which is compatible with a decaying particle (7), coming from the same vertex. The predicted trajectories are indicated in fig. $14 \mathrm{a}$, and the vertex can only be determined to a few $\mathrm{cm}$ in the beam direction. It should be noted that a better reconstruction is obtained in the horizontal plane, due to the small inclination of the wires in the drift chambers. Because fig. 14 is much enlarged compared to the distance of the drift chambers, a slight change in angle of the reconstructed track corresponds in the figure to a parallel shift of the track only. Therefore, a parallel shift can be applied to these tracks, in order to fit them to the information provided by the microstrip detector. In fig. $14 \mathrm{~b}$ the result thus obtained is shown. All shifts are compatible with the expected uncertainties. A considerable improvement is achieved in the vertex determination, using only a single microstrip plane.

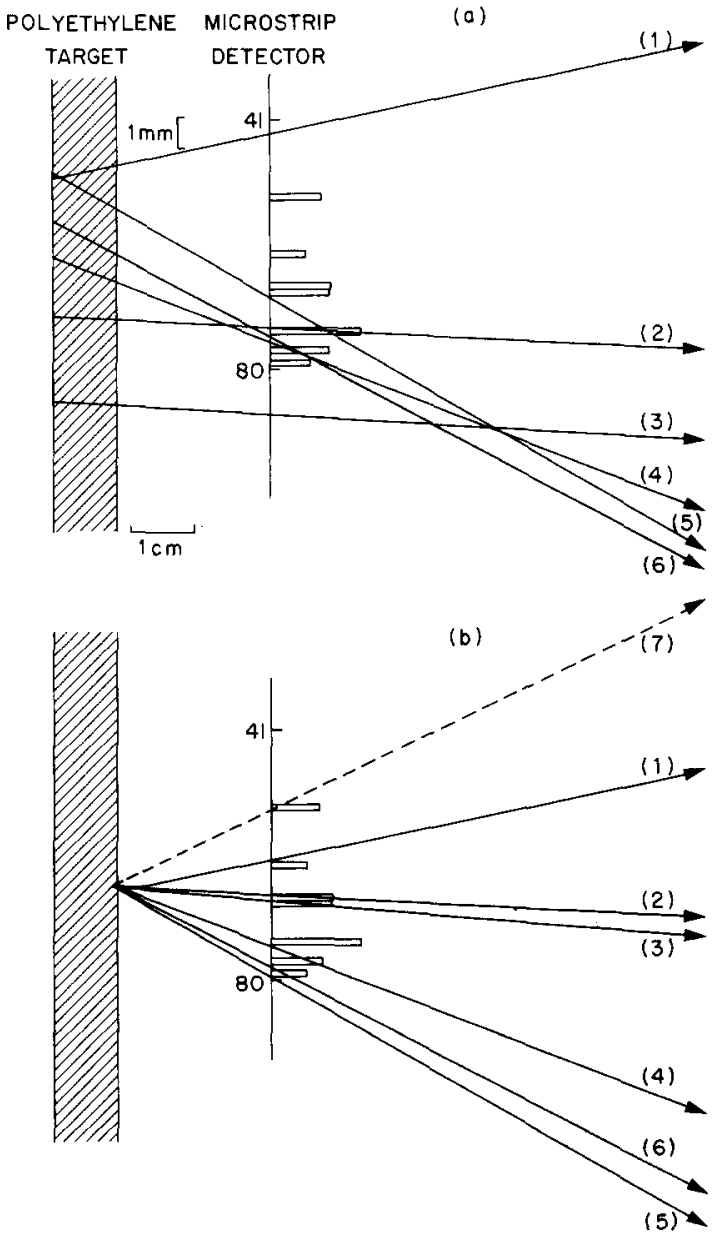

Fig. 14. (a) Event reconstructed in the vertical plane using drift chambers, placed at $3 \mathrm{~m}$ downstream. For the microstrip detector the corresponding pulse heights are indicated. (b) Microstrip detector information included in the reconstruction. Track (7) goes to the space point from where originate two tracks which are observed in the drift chambers. 


\section{Radiation damage}

The radiation damage to the silicon renders some applications of the microstrip detector unpractical or very expensive. One would like to know in advance the useful lifetime of the device rather precisely. But this is only possible if one knows exactly all the constituents of the radiation and their effect on the actually used silicon. Generally, the effort needed for this study is not worthwhile, and one can accept the lifetime, roughly predicted according to previous experience in analogous situations.

One should take into account the radiation damage parameters in the total design of a microstrip detection system. First of all, the resistivity of the silicon should be as low as possible, and p-type $\mathrm{Si}$ should be preferred. The reason is that the detector behaviour becomes critical if the number of introduced damage centres equals the number of electrically active doping atoms. For $20000 \Omega \cdot \mathrm{cm}$ n-type Si this number is only $5 \times 10^{11} \mathrm{~cm}^{-3}$, but for 1000 $\Omega \cdot \mathrm{cm}$ p-type it is $2 \times 10^{13} \mathrm{~cm}^{-3}$. Also most damage centers are acceptor type, and these influence less the p-type material: The high energy particles seem to introduce mostly simple defects, e.g. divacancies, in the very pure silicon which is normally used for detectors. The introduction rate for $\sim 2 \mathrm{GeV}$ muons was found to be of the order of $0.2 \mathrm{~cm}^{-3}$ per particle $\mathrm{cm}^{-2}$ [17]. An abundance of slow neutrons is more likely to be the limiting factor than the flux of high energy particles themselves. These neutrons cause large defect clusters, and $10^{11}$ thermal neutrons $\mathrm{cm}^{-2}$ seriously degrade detector operation. The defects cause an increase of detector leakage current, and consequently noise. Trapping and detrapping of charge, responsible for shortening of the minority carrier lifetime, deteriorate the signal. A fast, current sensitive preamplifier presents an advantage in this respect, because low frequency components of the noise are suppressed.

Altogether, the thinner the detector, the longer its radiation life because lower resistivity silicon can be used for a totally depleted volume, and because shorter signals can be obtained so that a reduced carrier lifetime has less influence.

One of our microstrip detectors received up to now a dose of $\sim 10^{12} \mathrm{~cm}^{-2}$ and no sign of degradation in performance is seen. In view of our previous experience with silicon detectors in high energy particle fluxes, we expect a useful life between $10^{13}$ and $10^{15}$ hadrons $\mathrm{cm}^{-2}$ depending on the abundance of slow neutrons and nuclear fragments from interactions.

\section{Applications of the silicon microstrip detector}

The starting point for the development of this microstrip detector was the emerging need for a thin detector, for high multiplicity which can be placed immediately behind a target (possibly a nuclear emulsion). By combination of several slices it can also be used as a high precision vertex detector. Because of its limited size, only applications at very high energy can be envisaged, and at rather short distance from the interaction region. The ultimate spatial resolution will be determined by the attainable detector thickness as well as the pitch of the strips. The very good double track resolution and the high rate capability may prove more valuable than the spatial resolution alone. On a single strip the particles are well separated in time up to a rate of $\sim 10^{6} \mathrm{~s}^{-1}$. For a 20 $\mu \mathrm{m}$ pitch, with strips of $20 \mathrm{~mm}$ long $\left(0.4 \mathrm{~mm}^{2}\right)$ a total rate in excess of $10^{8} \mathrm{~cm}^{-2} \mathrm{~s}^{-1}$ will not present a problem for the detector. An obvious application is therefore as beam profile monitor in a high intensity secondary beam. It can be used also as beam hodoscope but problems arise when coincidences have to be determined between signals from several detectors at rates of $10^{8}-10^{9} \mathrm{~s}^{-1}$.

The fact that the microstrip detector is thin compared to other detectors, is advantageous for the reconstruction of trajectories, especially in a magnetic field, where the microstrip detector can be operated without problem. But the position information will be degraded for small strips due to the Hall effect which causes "cross talk" and noise. In a field of a few $T$, the electrons in the silicon can have a deviation of $1 / 10$ of their path length.

Finally, the silicon microstrip detector can be used inside the vacuum chamber of a storage ring e.g. for elastic cross section measurements at small angles. The realization of such an application requires appreciable engineering, and detectors produced by diffusion or ion-implantation should be preferred because they can withstand a moderate bake-out.

\section{Conclusion}

In two separate test experiments we demonstrated that a silicon microstrip detector with a fast current 
preamplifier can easily be used with already existing electronics and read-out. It behaves from the user's point of view like a MWPC, but has much better spatial and time resolution for multiple track events and can stand rates in excess of $10^{7} \mathrm{~cm}^{-2} \mathrm{~s}^{-1}$. The efficiency is better than $99.6 \%$. The signals either on a single strip or on several strips, are proportional to the energy deposited in the detector. The area covered by one detector can be $5-70 \mathrm{~cm}^{2}$ but is limited by fan-out problems for very small strips. The thickness can be $400 \mu \mathrm{m}$ of $\mathrm{Si}$, and very close stacking of several detectors is possible. The detectors which we built and tested have a pitch of $200 \mu \mathrm{m}$. In following designs we envisage to use a pitch of $50 \mu \mathrm{m}$ and $20 \mu \mathrm{m}$. At the same time a reduction in size of the electronics has to be realized.

We would like to thank Mr. P. Burger of Enertec/ Schlumberger for the efficient co-operation in producing the detectors. The work of A. Gandi, J. Guerin, C. Nichols and A. Braem was highly appreciated. Staff of the EF Neutrino Beam Group put a big effort in achieving the installation in time.

Stimulating discussions with P. Siffert, F. Goulding and T. Ludlam are gratefully acknowledged.

\section{References}

[1] G. Bellini et al., Nucl. Instr. and Meth. 107 (1973) 85 and status report experiment NA1 CERN/SPSC/79-112.

[2] L.C.L. Yuan, Proc. Int. Conf. on Instrumentation for high energy physics, Berkeley (1960) p. 177.

[3] H. Esbensen et al., Phys. Rev. B18 (1978) 1039.

[4] P. Musset et al., experiment NA19, CERN/SPSC/79102.

[5] G. Diambrini-Palazzi et al., proposal P137, CERN/ SPSC/P137.

[6] J.S. Fleming, Nucl. Instr. and Meth. 150 (1978) 417.

[7] J.E. Bateman and W.R. Hogg, Nucl. Instr. and Meth. 79 (1970) 134.

[8] N.J. Hansen et al., Nucl. Instr. and Meth. 105 (1972) 293.

[9] J.E. Lamport et al., Nucl. Instr. and Meth. 134 (1976) 71.

[10] E.L. Haase et al., Nucl. Instr. and Meth. 97 (1971) 465.

[11] W.K. Hofker et al., IEEE Trans. Nucl. Sci. NS-13 (1966) 208.

[12] J.A. den Boer et al., Nucl. Instr. and Meth. 92 (1971) 173.

[13] E. Laegsgaard, Nucl. Instr. and Meth. 162 (1979) 93.

[14] V. Radeka and R.A. Boie, IEEE Trans. Nucl. Sci. NS27 (1980) 351.

[15] E.H.M. Heijne et al., IEEE Trans. Nucl. Sci. NS-27 (1980) 272.

[16] G. Charpak et al., Nucl. Instr. and Meth. 167 (1979) 455.

[17] H.M. Heijne et al., Rad. Eff. 29 (1976) 25. 